\title{
Subsurface diagnostics of quasi-one-dimensional inhomogeneities using the method of near-field microwave sounding
}

\author{
D. V. Yanin, A. G. Galka, A. V. Kostrov, A.I. Smirnov
}

Federal Research Center Institute of Applied Physics of the Russian Academy of Sciences (IAP RAS), Nizhny Novgorod, Russian Federation, dyanin@appl.sci-nnov.ru

Potentialities of the near-field microwave sounding are studied for the case of model media with spatially localized inhomogeneities.

The method of resonance near-field sounding [1$3]$ is used successfully to study electrodynamic properties of various substances in the microwave range. Depending on the operating frequency, either oscillatory circuits with lumped parameters or distributed resonance systems can be used as measurement sensors. When a sample is introduced into the region occupied by the quasi-static field of a sensor, its resonance frequency and the Q-factor change. These variations can be used to judge about the electric and magnetic properties of the studied object. Using the sensors with different sounding depths, one can study the internal structure of the object without disrupting its integrity.

The near-field measuring system, which we use for diagnostics of inhomogeneous media, was a microwave resonator in the form of a section of a loop line closed on one end. A measuring capacity at the other end of this section is made up by two parallel wires with the radius $r=0.5 \mathrm{~mm}$, the length $\mathrm{d}_{\mathrm{m}}=4 \mathrm{~cm}$, and the varying distance $d$ between the wires. Magnetic coupling loops were used to excite the resonator and receive its responsse. In this case, the maximum sounding depth was close to $d$. Eight sensors with sounding depths ranging from $5 \mathrm{~mm}$ to $25 \mathrm{~mm}$ were used to study the internal structure of the media. The eigen frequencies $f_{0}$ of the measuring systems were about $550 \mathrm{MHz}$, and their $Q$-factors, about 150 .

The relationship between the resonant frequency $f$ of the sensor and the parameters of the inhomogeneous medium is expressed as follows

$$
\frac{f-f_{0}}{f_{0}}=\frac{d}{l}\left(1-\varepsilon_{\text {int }} \frac{\rho_{1}}{\rho_{2}}\right)
$$

where $\varepsilon_{\text {int }}$ - the integral permittivity of the inhomogeneous medium, $\rho_{1}$ and $\rho_{2}$ - wave resistances of twowire lines corresponding to the resonator and the measuring capacitance, $l$ - the length of the resonator. In the derivation of the expression (1) it was assumed that the electrical length of the measuring part is significantly less than the wavelength, $d_{m}<<c /\left(f_{0} \varepsilon_{\text {int }}\right)$ ( $c$ - the speed of light in vacuum). In the case of planar media, using the image method, you can obtain the following expression for $\varepsilon_{\text {int }}$.

$$
\varepsilon_{\mathrm{int}}=\left(\varepsilon_{1}+1\right)\left(2+\left(\frac{2 \varepsilon_{1}}{\varepsilon_{1}+1}\right)\left(\frac{\varepsilon_{1}-\varepsilon_{2}}{\varepsilon_{1}+\varepsilon_{2}}\right) \frac{\ln \left(\frac{d^{2}+(2 h)^{2}}{r^{2}+(2 h)^{2}}\right)}{\ln \left(\frac{d}{r}\right)}\right)^{-1}
$$

$h$ - depth of inhomogeneity, $\varepsilon_{1}$ and $\varepsilon_{2}$-dielectric permeability of the medium and inhomogeneity, respectively.

The medium studied in the experiments, in which the method of near-field microwave sounding was tested, was organic glass having the dielectric permittivity $\varepsilon_{1}=3.5$ with the rectangular inclusions of glass having the dielectric permittivity $\varepsilon_{2}=7.8$, which were situated at the depths $\mathrm{h}=4$ and $5.5 \mathrm{~mm}$. The length and thickness of the inhomogeneity were equal to $L_{n}=10.5$ $\mathrm{cm}$ and $\mathrm{h}_{\mathrm{n}}=7 \mathrm{~cm}$, respectively.

To spot the inhomogeneities, a probe with a sounding depth of $10 \mathrm{~mm}$ moved parallel to the flat surface of the organic glass. The value of the shift of the resonance frequency $\Delta \mathrm{f}$ was registered depending on the position $x$ of the sensor (see Fig.1).

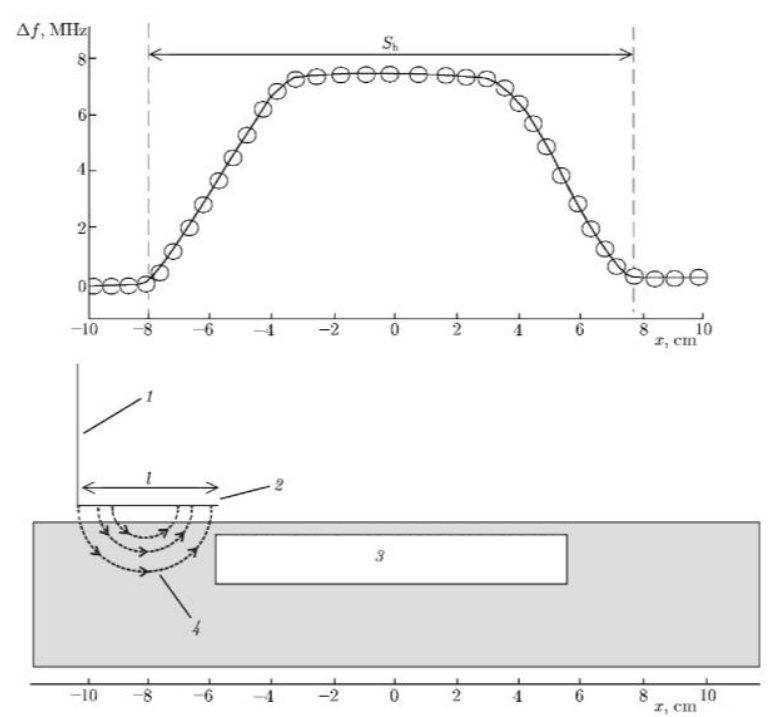

Fig. 1. Shift of the resonance frequency $\Delta f$ of the sensor with a sounding depth of $10 \mathrm{~mm}$ as a function of the probe coordinate $x$ for the case of scanning along the plane surface of the medium with a rectangular inhomogeneity.

The length of inhomogeneities is equal to the difference between the length s of the "swell" in the plot of the resonance frequency and the length $d$ of the wires of the measuring capacity.

Further, the medium was studied using sensors with different sounding depths. The resonance frequency of the measuring systems was registered as the systems contacted the surface of the medium near the center of the inhomogeneity. Then, the dielectric permittivity $\varepsilon_{\mathrm{ex}}$ of the medium was determined for each probe using Eq. (1).

$F\left(\varepsilon_{1}, \varepsilon_{2}, h\right)=\sqrt{\frac{1}{n} \sum_{i=1}^{n}\left(\varepsilon^{e x}\left(d_{i}\right)-\varepsilon_{\mathrm{int}}\left(\varepsilon_{1}, \varepsilon_{2}, h, d_{i}\right)\right)^{2}}$ 
To measure the medium parameters, we minimized function (3) being the r.m.s. deviation between $\varepsilon_{\text {int }}\left(\varepsilon_{1}, \varepsilon_{2}, \mathrm{~h}, \mathrm{~d}\right)$ for the plane-layered medium and the experimentally found values of the dielectric permittivity $\varepsilon_{\text {ex. }}$. Table shows the actual and experimental values of the electrodynamic and geometric parameters of the studied media.

This paper deals with testing of the method of the near-field microwave sounding. The algorithm of solving of the inverse problem is presented, and the geometric and electrodynamic parameters of rectangular inhomogeneities are determined with good accuracy.

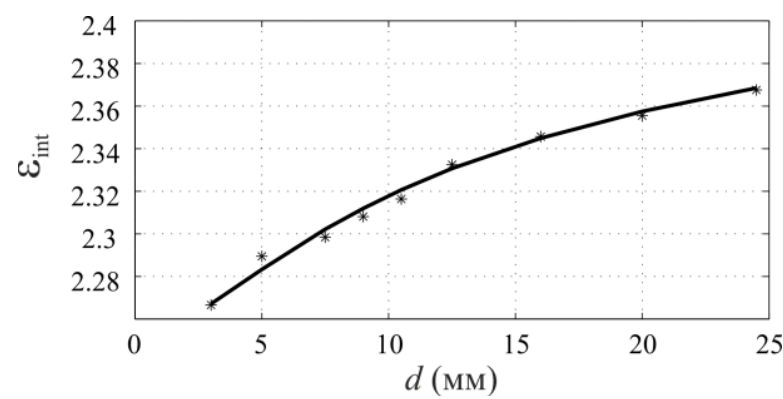

Fig. 2. Integral dielectric permittivity $\varepsilon_{\text {int }}$ as a function of the distance $d$ between the wires of the sensor measuring head in the case of studying an inhomogeneity in the form of a rectangular parallelepiped. The asterisks mark the experimental data, and the solid curve represents the theoreti- cal dependence that corresponds to Eq. (2) for the found values of $\varepsilon_{1}, \varepsilon_{2}$ and h.

\begin{tabular}{|c|l|l|l|l|}
\hline Model media 1 & $\varepsilon_{1}$ & $\varepsilon_{2}$ & $h$ & $L_{n}$ \\
\hline Experiment & 3.5 & 7.3 & 5.0 & 11.4 \\
\hline Real value & 3.5 & 7.8 & 5.5 & 10.5 \\
\hline & & & & \\
\hline Model media 2 & $\varepsilon_{1}$ & $\varepsilon_{2}$ & $h$ & $L_{n}$ \\
\hline Experiment & 3.5 & 7.6 & 3.6 & 11.4 \\
\hline Real value & 3.5 & 7.8 & 4.0 & 10.5 \\
\hline
\end{tabular}

This work was supported by the Russian Foundation for Basic Research (project Nos. 18-42-520053 p_a).

\section{References}

1. D. V. Yanin, A. V. Kostrov, A. I. Smirnov, et al. Diagnostics of the Atmospheric Pressure Plasma Parameters Based on Near-Field Microwave Plasma Probing // Tech. Phys., 82, No. 4, 468 (2012).

2. Yanin D.V., Galka A.G., Smirnov A.I., Kostrov A.V., Strikovskii A.V. Resonant near-field microwave diagnostics of inhomogeneous mediums // Advances in Applied Physics. 2014, 2, No. 6. P. 555-570.

3. Martusevich A.K., Galka A.G., Krasnova S.Yu., Yanin D.V., Bogomolova E.B. Study of dielectric properties of human skin: the first experience of microwave probing. International Journal Of Applied And Fundamental Research. - 2017. - № 3 